\title{
Development of an International Canine Spinal Cord Injury observational registry: a collaborative data-sharing network to optimize translational studies of $\mathrm{SCl}$
}

\author{
Sarah A. Moore ${ }^{1}$ Natalia Zidan ${ }^{2}$ Ingo Spitzbarth ${ }^{3,4} \cdot$ Yvette S. Nout-Lomas $^{5}$ - Nicolas Granger ${ }^{6}$. \\ Ronaldo C. da Costa ${ }^{1} \cdot$ Jonathan M. Levine ${ }^{7}$ Nick D. Jeffery ${ }^{7} \cdot$ Veronika M. Stein $^{8} \cdot$ Andrea Tipold $^{4,9}$. \\ Natasha J. Olby ${ }^{2,10}$
}

Received: 30 January 2018 / Revised: 23 March 2018 / Accepted: 9 April 2018 / Published online: 23 May 2018

(c) International Spinal Cord Society 2018

\begin{abstract}
Study design Prospective cross-sectional cohort study.

Objectives The canine spontaneous model of spinal cord injury (SCI) is as an important pre-clinical platform as it recapitulates key facets of human injury in a naturally occurring context. The establishment of an observational canine SCI registry constitutes a key step in performing epidemiologic studies and assessing the impact of therapeutic strategies to enhance translational research. Further, accumulating information on dogs with SCI may contribute to current "big data" approaches to enhance understanding of the disease using heterogeneous multi-institutional, multi-species datasets from both pre-clinical and human studies.

Setting Multiple veterinary academic institutions across the United States and Europe.

Methods Common data elements recommended for experimental and human SCI studies were reviewed and adapted for use in a web-based registry, to which all dogs presenting to member veterinary tertiary care facilities were prospectively entered over $\sim 1$ year.

Results Analysis of data accumulated during the first year of the registry suggests that $16 \%$ of dogs with SCI present with severe, sensorimotor-complete injury and that $15 \%$ of cases are seen by a tertiary care facility within $8 \mathrm{~h}$ of injury. Similar to the human SCI population, $34 \%$ were either overweight or obese.

Conclusions Severity of injury and timing of presentation suggests that neuroprotective studies using the canine clinical model could be conducted efficiently using a multi-institutional approach. Additionally, pet dogs with SCI experience similar comorbidities to people with SCI, in particular obesity, and could serve as an important model to evaluate the effects of this condition.
\end{abstract}

\section{Introduction}

Client-owned pet dogs commonly experience spontaneous spinal cord injury (SCI) caused by acute intervertebral disc herniation (IVDH), with 20,000-30,000 new cases managed by veterinary spinal surgeons in the United States alone each year [1]. Certain chondrodystrophoid breeds of dog,

Electronic supplementary material The online version of this article (https://doi.org/10.1038/s41393-018-0145-4) contains supplementary material, which is available to authorized users.

Sarah A. Moore

moore.2204@osu.edu

Extended author information available on the last page of the article such as the dachshund, Pekingese, and others have a genetic predisposition to degeneration of the intervertebral disc. An expressed fibroblast growth factor (FGF)-4 retrogene has been associated with chondrodyplasia and intervertebral disc degeneration in these dog breeds [2]. Intervertebral disc degeneration in turn predisposes the disc to herniate into the vertebral canal, subsequently producing a mixed contusive/ compressive injury to the respective spinal cord segments [3]. Severity of neurologic injury by this mechanism spans a range of clinical signs from pain and mild weakness up to sensorimotor-complete SCI. Patterns of sensory and locomotor recovery are predictable and well-documented for different injury severities [4, 5]. IVDH-associated SCI can occur in either the cervical or thoracolumbar spinal cord, but thoracolumbar SCI is the most common 
presentation, providing a plentiful and convenient clinical model of SCI for study.

Behavioral assessments commonly used in the veterinary clinical setting to evaluate dogs with IVDH-associated SCI include several ordinal locomotor scales validated for use in dogs, the assessment of proprioceptive responses and spinal reflexes, subjective evaluation of the animal's behavioral response to a noxious stimulus (used as a surrogate assessment of normal, diminished, or absent nociceptive processing), and a variety of quantitative sensory testing methods [6-11]. For spinal cord lesions affecting the thoracic or lumbar segments, lesion level can be determined based on the presence or absence of segmental spinal reflexes in the pelvic limbs (spinal shock is extremely shortlived in dogs) and whether a "cutoff" of the cutaneous trunci reflex exists. For dogs with moderate and severe thoracolumbar SCI, the cutaneous trunci reflex is typically absent beginning approximately two vertebral segments caudal to the lesion [12]. Following clinical examination, dogs often undergo advanced imaging such as computed tomography or magnetic resonance imaging for additional lesion definition as well as for purposes of surgical planning.

Dogs with clinical SCI represent a large animal model that offers unique benefits for SCI research. This includes heterogeneity of both injury and genetic backgrounds and histopathologic similarities to those lesions observed in people, altogether providing the ability to conduct veterinary clinical trials in a setting that closely mirrors intention to treat/interventional trials in people [13-17]. While a large cohort of SCI-affected dogs are managed across tertiary care veterinary hospitals each year, a much smaller number are cared for at any single given facility. Thus, the establishment of an international observational veterinary patient registry constitutes a key step in performing epidemiologic studies and assessing the impact of therapeutic strategies to facilitate translational research. A harmonized approach to data collection across researchers using the canine clinical model is much needed, and can serve to streamline information while maximizing comparisons across studies and tertiary care facilities caring for dogs with SCI. Further, accumulating information on clinical SCI in dogs may contribute to current "big data" approaches to enhance understanding of SCI using heterogeneous multi-institutional, multi-species datasets from both pre-clinical and human clinical SCI $[18,19]$. It may contribute to datasharing approaches, which have been endorsed by groups such as the Office of Data Science at the NIH [20]. Here, we provide rationale and framework for the Canine Spinal Cord Injury (CSCI) observational registry and share our experience in the establishment of what, to our knowledge, is the first international registry for canine patients with SCI. We also present preliminary data related to demographics, descriptive statistics, and future directions for advancing translational research in SCI using information accumulated from the CSCI observational registry.

\section{Methods}

\section{Summary of the establishment of CANSORT-SCI}

The Canine Spinal Cord Injury Consortium (CANSORT$\mathrm{SCI})$ was established as a research consortium in November 2015, at a meeting organized by Texas A\&M University and supported by the Mission Connect Foundation [16]. The CANSORT-SCI network is a collaboration of veterinary neurologists and neurosurgeons with training in neuroscience, veterinary pathologists, and basic neuroscience researchers motivated to conduct leading edge clinical research in the field of SCI using the spontaneous canine model. The network currently encompasses seven veterinary institutions in the United States and Europe. One of the primary goals identified by the group at its initial meeting was the establishment of a canine SCI "patient" registry and data repository to serve as a collaborative collection of comprehensive clinical data by which to facilitate future clinical trials and epidemiologic studies.

\section{Selection of common data elements}

The establishment of the CSCI registry was prioritized as an early project by the group, with the purpose of including relevant demographic, clinical, and biochemical data for dogs with natural SCI. The intention was to develop an observational, longitudinal veterinary patient registry, which was readily available to all CANSORT-SCI network members for prospective collection of standardized case information. To allow for inclusion of uniform data, standardized electronic case report forms were developed by the CANSORT-SCI network after review of common data elements (CDEs) proposed for collection in both experimental animal models and human SCI [21-23]. Because the canine model of SCI represents a hybrid animal model that is spontaneous and managed in a veterinary clinical setting, a combination of elements from both the experimental and clinical CDEs were selected by expert opinion to be both relevant and applicable to this unique model. Specific data related to medical history, physical, and neurological examination at the time of diagnosis, demographics, results of biochemical and imaging diagnostics, treatment modalities (including surgery and drug administration) were entered into an online form. Additionally, information about the availability and type of samples banked for each animal, including blood, cerebrospinal fluid, and tissue for 
Table 1 A modified version of the Frankel scale used for scoring sensorimotor status of dogs with acute intervertebral disc herniation-associated spinal cord injury of the thoracolumbar spine

\begin{tabular}{ll}
\hline Grade & Description \\
\hline 0 & $\begin{array}{l}\text { Paraplegia with absent superficial and deep pain sensation; there is an absence of behavioral } \\
\text { response (e.g., vocalizing or orienting movement of the head toward stimulus) when clamping of a } \\
\text { hemostat or other instrument to the skin of the limb/paw (superficial) and when applying the same } \\
\text { stimulus while clamping the bone of the digit (deep). There is no observable movement of the hind } \\
\text { limbs } \\
\text { Paraplegia with absent superficial but intact deep pain sensation; behavioral response (e.g., } \\
\text { vocalizing or orienting movement of the head toward stimulus) is absent when clamping the skin } \\
\text { (superficial) but present when clamping the bone of the digit (deep). There is no observable } \\
\text { movement of the hind limbs } \\
\text { Paraplegia with intact superficial and deep pain sensation; there is presence of a behavioral } \\
\text { response (e.g., vocalizing or orienting movement of the head toward stimulus) to both superficial } \\
\text { and deep noxious stimuli. There is no observable movement of the hind limbs } \\
\text { Non-ambulatory paraparesis; there is movement of one or both hind limbs, but the animal is unable } \\
\text { to take 10 consecutive unassisted weight-bearing steps } \\
\text { Ambulatory paraparesis; the animal can take 10 consecutive unassisted weight-bearing steps with } \\
\text { the hind limbs but displays an ataxic or paretic gait } \\
\text { Paraspinal hyperesthesia only; the animal has a normal hind limb gait but has posture or physical } \\
\text { examination findings indicative of paraspinal hyperesthesia }\end{array}$ \\
Normal
\end{tabular}

histopathology if applicable were are also entered (Supplemental material - data collection form)

\section{Eligibility criteria}

All dogs that are presented to a CANSORT-SCI member institution with an acute SCI are enrolled regardless of ultimate cause identified. This approach is intentionally broad to allow the enrollment of not only cases with the most common cause of acute SCI in dogs, IVDH-associated SCI, but also to encourage data collection for less common causes of SCI in dogs that may also have translational relevance such as fibrocartilaginous embolic myelopathy/ ischemic myelopathy, and extrinsic spinal trauma such as motor vehicle accidents and falls (fracture/luxation).

\section{Clinical evaluation of dogs with spinal cord injury}

Veterinarians and researchers in canine clinical SCI rely heavily on categorical and ordinal grading schemes to describe severity of injury and recovery of dogs with thoracolumbar SCI. An assortment of scoring systems are used in canine SCI literature, including the modified Frankel scale [6], Tarlov scale [24], Texas Spinal Cord Injury Scale (TSCIS) [8], the Open Field Score [7], and the modified canine Basso-Beattie-Bresnahan locomotor rating scale (canine BBB) [9]. A future goal of CANSORT-SCI is to evaluate the comparative utility of these scoring systems and to develop a unified approach to locomotor and sensory assessment across testing centers; therefore data were collected for each dog with respect to multiple clinical grading scales using previously published methods and definitions.
However, in the most basic clinical categorization, dogs are pronounced ambulatory vs. non-ambulatory, where ambulatory indicates that the animal can bear weight consistently in both pelvic limbs without additional external support for 10 consecutive steps [16]; as having the presence or absence of motor function (is any voluntary movement noted at all in the affected limbs?); and as having the presence or absence of a behavioral response to a nociceptive stimulus such as clamping the digit with a hemostat or other instrument. For the purposes of initial data reporting related to the CSCI, dogs were categorized using a modification of the Frankel scale that is commonly employed by veterinarians in the clinical setting and encompasses these basic categorical factors. The scale ranges from 0 to 6 with a grade of zero representing a sensorimotor-complete lesion and a grade of 6 representing a neurologically normal dog (Table 1).

\section{Ethical considerations}

The CSCI observational registry complies with all applicable local regulations relating to ethical treatment of animals in research and veterinary patients in clinical studies. No individual patient identifiers are included on the data collection forms. The patient's location of enrollment and electronic medical record number are entered in to the database at the time an initial record is generated and immediately linked to a unique CSCI registry number, which is then associated with that patient's data record. In all participating countries, informed consent is not mandatory for prospective data collection from veterinary patients, assuming data collected is passive/observational only and 
treatment does not deviate from "standard of care." All aggregated data or results provided from the registered data are strictly anonymous.

\section{Data collection}

Member institutions of the CANSORT-SCI network are committed to delivering data to the CSCI registry for all eligible cases treated at their respective hospital. For each new patient entered, a minimum data set is required (Table 1) including demographic information and SCI etiology. Each patient entered in to the registry is assigned a unique identifier (alphanumeric code). Clinical data are directly collected in a standardized format by the clinician. A resource person-a CANSORT-SCI site PI or clinical research assistant, depending on the site-is responsible for entry of data in to the database. The CSCI registry CDEs are organized in to five categories related to clinical history, patient demographics, examination findings, diagnostic work-up, and treatment. Future directions include the development of longitudinal follow-up forms to document long-term patient outcomes. A major concern regarding feasibility of the project was time required per case for data entry. Therefore, a time stamp was added to the beginning and end of the survey form so that resource personnel could indicate the total amount of time required to enter data for each individual animal.

\section{Data storage and safety}

Electronic data collection forms were generated and study data were collected and managed using REDCap electronic data capture tools (www.project-redcap.org) hosted at The Ohio State University [25]. REDCap (Research Electronic Data Capture) is a secure, data capture tool, which is designed to provide an intuitive, web-based interface to support data capture for research studies. Important features include the ability to track data export and manipulation; automated export procedures that allow data download for commonly used statistical packages; and the ability to import data from external sources. REDCap provides several active protective features to prevent unsolicited use of the application including user/role validation. An audit trail module allows tracking of all accesses, modifications, and deletions of the data.

Data collection began in the form of beta testing at a single CANSORT-SCI institution from June through August 2016, during which time all dogs presenting only with IVDH-associated SCI were entered in to the registry using the pre-developed data collection instrument. During this time, data was entered by a single user who had not been involved in development of the data collection instrument (N.Z.). This user made detailed observations related to clarity of the instrument, ease of data entry, time involved in data entry for each patient, and noted any opportunities for improvement of the process or the instrument itself. During this beta testing phase, small improvements were made in descriptors related to injury severity, additional questions were added to the instrument focusing on diagnostic certainty, and criteria were expanded to include causes of SCI beyond IVDH. The project was moved from testing to production phase in Fall 2016, at which time staged on-boarding of other CANSORT-SCI member institutions began.

\section{Results}

\section{Data entry}

Over the following 12-month period, 181 cases were entered from a total of five institutions. Because of staged on-boarding of data entry institutions and individual limitations with respect to resource personnel, the remaining 2 CANSORT-SCI institutions anticipate commencing data entry within the next year. Median time recorded for data entry of an individual case record was $6 \mathrm{~min}$ (range $2 \mathrm{~min}$ to $3 \mathrm{~h}$ and $47 \mathrm{~min}$; mode $5 \mathrm{~min}$ ). Ninety percent of cases required $15 \mathrm{~min}$ or less of total data entry time.

\section{Demographic data}

Median age for cases entered in the database was 6 years (range 1-13 years) and $90 \%$ of cases weighed $\leq 20 \mathrm{~kg}$ (range $3-50 \mathrm{~kg}$ ) at the time of hospital admission. Spayed female and castrated male dogs were similarly represented (Fig. 1). Breeds for which five or more cases were represented included dachshund $(n=62)$, French bulldog $(n=21)$, mixed breed $\operatorname{dog}(n=16)$, beagle $(n=10)$, Cocker spaniel $(n=6)$, and shih tzu $(n=5)$. The majority of dogs entered in to the database were considered by their attending clinician to be systemically healthy at the time of presentation apart from their SCI, with $84 \%$ listed as having no other significant health issues; however, $34 \%$ percent of dogs were listed as having a body condition score (BCS) of $\geq 4$ (scale range 1-5), suggesting they were either overweight or obese. Obesity was the most common clinician-reported comorbidity, affecting $7 \%$ of SCI-affected dogs currently included in the database, followed by orthopedic disease (unspecified; 4\%), and either presumptive or confirmed mitral valve endocardiosis (3\%).

\section{Etiology, duration, and severity of injury}

The neurological level, and severity of injury for all cases entered in the database are summarized in Fig. 2. Acute 
A

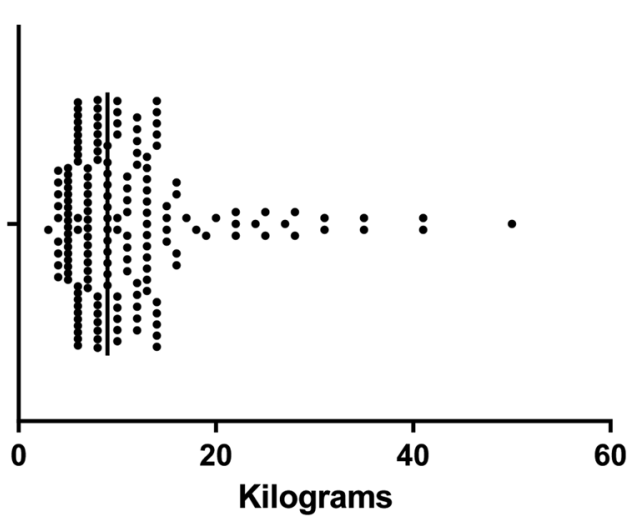

C

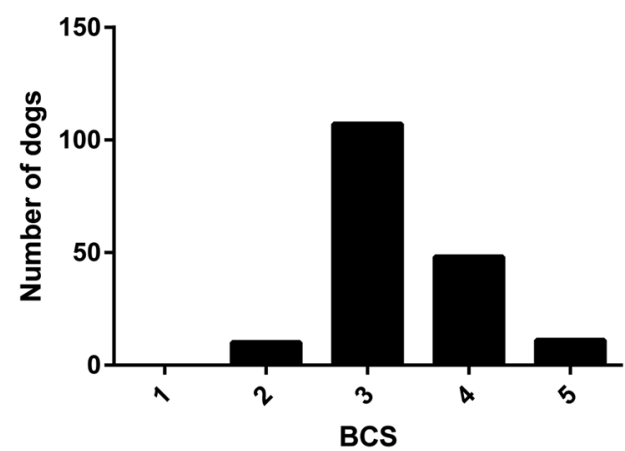

Fig. 1 Demographic information for dogs with clinical SCI entered into the CSCI patient registry between June 2016 and January 2018, including body weight in kilograms (a), sex (b), body condition score

intervertebral disc extrusion was the cause of SCI in all but three cases entered in to the database to date. These cases consisted of two acute non-compressive nucleus pulposus extrusions and one traumatic thoracolumbar fracturesubluxation. The most common neurological level of injury was between the third thoracic and third lumbar spinal cord segments, affecting $87 \%$ of dogs. For reference, dogs have 13 thoracic and 7 lumbar vertebrae and the termination of the spinal cord is usually at the L6-7 vertebral level. The most common injury severity on presentation was "non-ambulatory paraparesis" (motor function present in both hind limbs, but inability of the dog to walk unassisted for 10 or more consecutive steps). Sixteen percent of dogs were presented with sensorimotor-complete injuries.

Duration of injury, defined as when the owner first noted signs of neurologic dysfunction, prior to presentation at a CANSORT-SCI (secondary/specialty care) facility was known or could be reliably approximated in $94 \%$ of cases and ranged from $1 \mathrm{~h}$ to 120 days (median $24 \mathrm{~h}$ ) (Fig. 3). Fifteen percent of the total caseload was presented within 8 $\mathrm{h}$ of injury, $52 \%$ of the total caseload was presented within $24 \mathrm{~h}$ of injury, and $82 \%$ of the total caseload was presented within $72 \mathrm{~h}$ of injury.

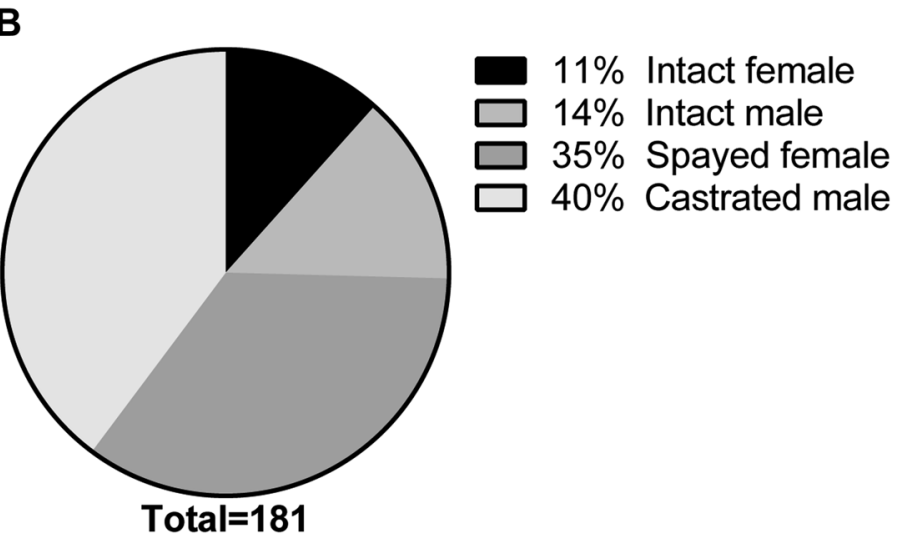

Age at presentation

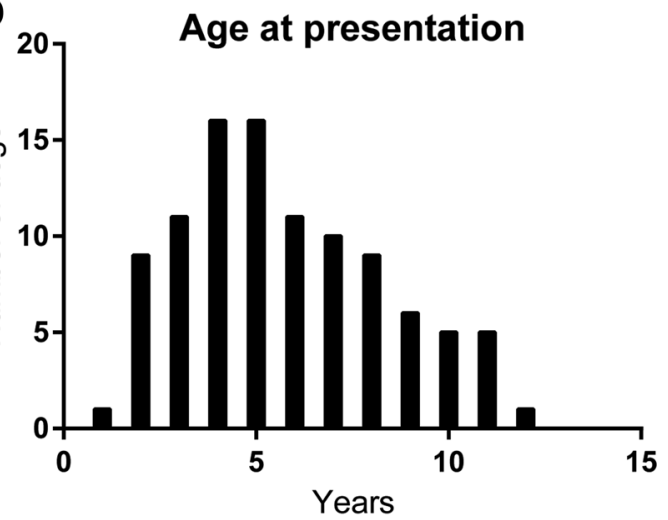

(ranging from 1-5; c), and age in years at the time of presentation for SCI (d). Line (a) corresponds to median value

\section{Treatments administered}

The electronic data form asks for information regarding corticosteroid, non-steroidal anti-inflammatory, and neuroprotective treatments administered both in a period of 7 days and 1 day prior to admission. The most common response at both time points was that the patient had received none of the above. Forty-nine percent and $41 \%$ of cases were naive to anti-inflammatory or neuroprotective treatments within a period of 7 days or $24 \mathrm{~h}$ prior to hospital admission, respectively. No dog received methylprednisolone sodium succinate (MPSS) prior to admission. Nineteen percent of dogs had received other (non-MPSS) corticosteroids at variable doses within 7 days prior to admission, $30 \%$ had received a non-steroidal anti-inflammatory medication, and $2 \%$ had received acetylsalicylic acid.

\section{Discussion}

The aim of this report was to describe the process of developing a novel international veterinary patient registry for neurologic disease, specifically SCI. The goals for 
Fig. 2 Neuroanatomic location (a) and severity of injury (b) for dogs with clinical SCI entered into the CSCI patient registry between June 2016 and January 2018. Between T3-L3

(anatomical equivalent of a midthoracic supra-sacral injury in a person) was the most common site of injury. Sixteen percent of dogs had sensorimotor-complete injuries at the time of presentation
A Neuroanatomic Location

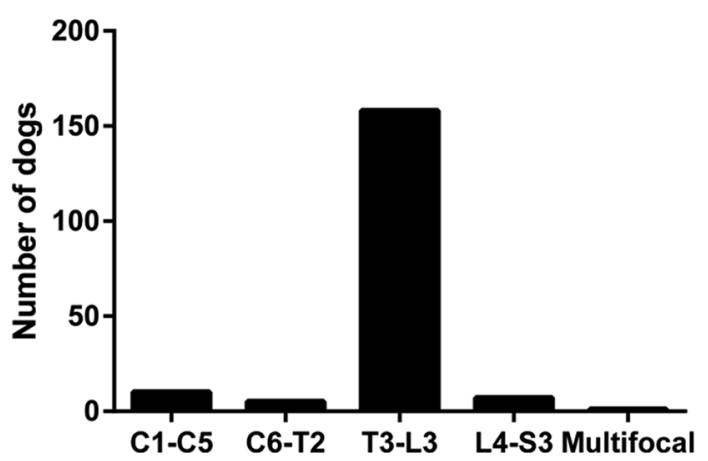

B

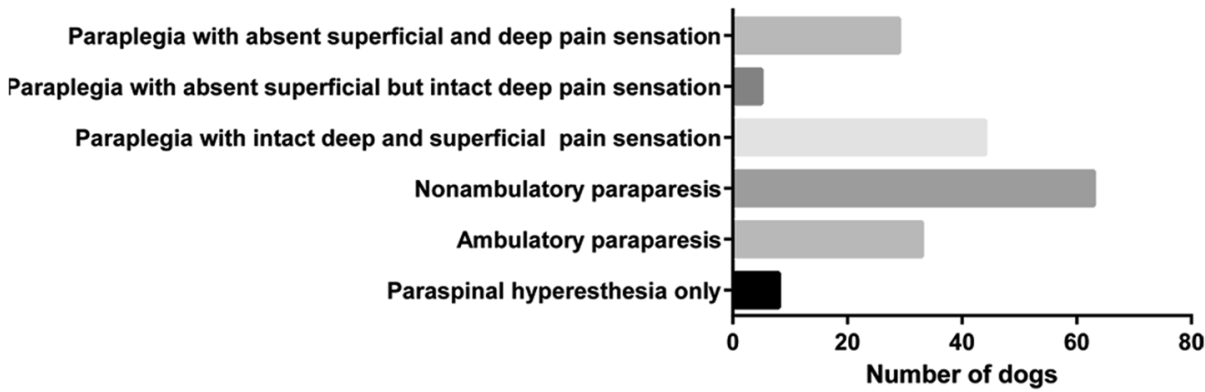

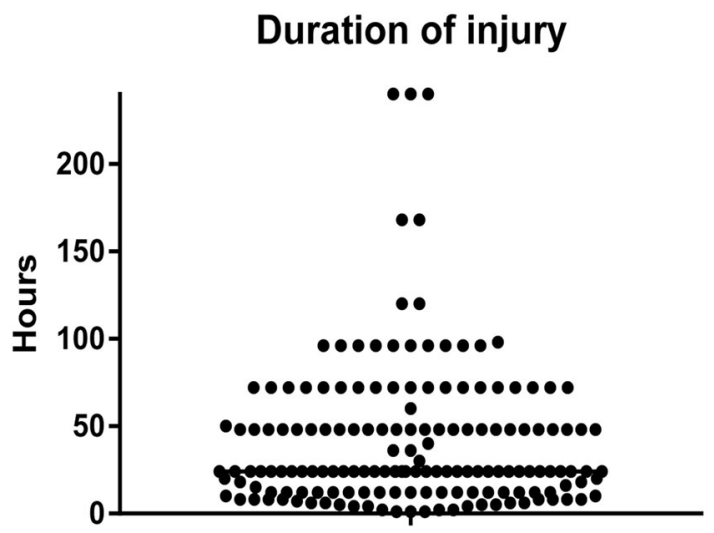

Fig. 3 Duration of injury, defined as when the owner first noted signs of neurologic dysfunction prior to presentation, for dogs with clinical SCI entered into the CSCI patient registry between June 2016 and January 2018. Duration of injury ranged from $1 \mathrm{~h}$ to 120 days (median $24 \mathrm{~h}$ ) and $15 \%$ of cases were presented within $8 \mathrm{~h}$ of injury. For ease in graphical representation dogs with injury duration $>240 \mathrm{~h}$ are not depicted $(n=12)$

creation of this registry are twofold: first to contribute to multi-species datasets that can lead to new discoveries and treatments of disease, and second to develop a large set of epidemiologic data specific to the dog, from which to draw conclusions and develop hypotheses for translational studies using the canine clinical model of SCI. While still early in the process of data collection, preliminary analysis of accumulated cases identifies several important demographic factors that may inform future translational studies using the canine clinical model of SCI.

The overall demographic data for dogs currently registered in the database parallels what has been previously reported in the literature with respect to IVDHassociated SCI, with young to middle-aged dachshunds and other chondrodystrophic breeds being overly represented [16]. Lesion localization in the present study is also in keeping with what is expected based on the veterinary literature, where injuries in the T3-L3 region of the spine are by far the most common [4, 5]. Assessment of comorbidities in dogs with SCI is of interest, with respect to how these may parallel the human SCI population where pre-existing problems such as obesity, cardiac disease, and other general health problems may influence response to particular therapies and recovery after injury $[26,27]$. Obesity was the most common comorbidity documented in the current study, with $34 \%$ of cases being either overweight or obese based on a common measure of obesity in dogs called the BCS. BCS is used in dogs similarly to body mass index in people, since conformational differences between breeds may influence what is an appropriate body weight for any given dog. The scale used in our database ranges from 1 to 5 with a score of 1 being emaciated, 3 being ideal body weight for the patient's confirmation, and 4 and 5 representing the patient being overweight to obese, or grossly obese, respectively [28]. The prevalence of obesity in this cohort 
of SCI-affected dogs is similar to that observed in the general canine population, where between 22 and $40 \%$ of dogs are reported to be obese, and also mirrors that seen in the general human population $[29,30]$ (https://www.cdc. gov/obesity/data/adult.html). Like in people, obesity in dogs results in increased circulating levels of proinflammatory cytokines, various other metabolic and hormonal impairments, and gut dysbiosis that could influence recovery after SCI [31-35]. The natural occurrence of these factors in the clinical dog population may make this model an ideal one for further exploring the detrimental effects of obesity on recovery and ways to improve outcome in this segment of the SCI population.

The prevalence of cardiac disease was low in the present study, effecting only $3 \%$ of dogs with SCI. When present, cardiac disease was attributed to either confirmed or presumptive mitral valve endocardiosis, a degenerative condition typified by myxomatous nodular changes to the mitral valve [36]. This condition is most commonly observed in small breed dogs and in severe cases can lead to profound mitral regurgitation, increasing chronic volume load to the left side of the heart, and in some cases congestive heart failure [37]. While prevalence of the number of dogs with heart disease in the current study is too low to assess relationships, future studies of continually accrued data may evaluate the association between cardiac status and outcome in dogs with SCI.

The most common cause of SCI in dogs in the current study was IVDH-associated SCI, which accounted for all but three of the cases entered in the CSCI. This is reflective, in part, of the fact that IVDH-associated SCI is noted to be the most common cause of SCI in dogs [38, 39]. The prevalence of other causes of SCI such as ischemic myelopathy and extrinsic trauma causing vertebral fracture/luxation is likely falsely low in the reported cohort of dogs because initial efforts related to the database focused only on accrual of IVDH cases. Data collection was only recently expanded to include ischemic myelopathy and vertebral fracture/ luxation, thus prevalence and other data related to these types of injuries will evolve over time as additional cases are accrued.

An interesting preliminary point based on information from the registry is the timing of presentation of dogs with SCI to a secondary care facility. This information will be useful for the planning of future studies using dogs with clinical SCI and suggests that a proportion of dogs (15\%) are presented within the peracute $(<8 \mathrm{~h})$ window that may be required for studies targeting acute neuroprotection. One caveat to this finding is that two institutions entering cases into the database during the study period were conducting veterinary clinical trials in SCI, which may have encouraged early referral of cases to their facility and influencing the number of cases presenting acutely and with more severe injuries; however, the apparent prevalence of sensorimotor-complete injuries in the current study is similar to what has been reported in other studies [39]. Accrual of additional cases from multiple institutions will be necessary to fully evaluate the proportion of dogs with acute, subacute, and chronic timing of presentation that may be available for clinical trials; however, preliminary data suggests that efficient acute neuroprotective studies should be feasible when a multi-institutional approach is used. Additionally, approximately half of cases were treatmentnaive with respect to neuroprotective treatments at the time of admission. This information can facilitate sample size determinations and their feasibility for future clinical studies of both neuroprotective strategies and other treatment interventions.

Initially, the CSCI registry collected information only about the initial presentation of a particular dog. Since that time, the development of follow-up forms has been a priority and a survey instrument to collect information about each dog during subsequent hospital visits has been drafted and will move into the beta testing phase shortly. An additional future direction for the registry is the generation of forms which might collect owner-derived quality of life information about dogs living chronically with SCI, and which could sporadically solicit electronically entered information directly from owners to track development of new comorbidities in dogs long term after injury.

One important goal of the registry is the inclusion of a "virtual tissue bank" function, where patient demographic, injury, and ultimately outcome information could be linked to biological samples, which could be made available for study by both member and non-member investigators. Most CANSORT member institutions currently have established biobanks for maintaining samples from dogs with SCIsome institutionally supported and some residing within individual laboratories. The survey instrument used for the registry asks the data entry person to indicate whether whole blood, serum, urine, or tissue are available from each individual case allowing the group to identify and track samples across multiple institutions. Inputted data is searchable such that investigators can request a list of cases for which a particular sample type is available, along with information regarding where those samples are stored. This approach will facilitate histopathological and molecular studies upon canine SCI, with the ultimate goal to increase the knowledge about morphologic and molecular alterations in naturally traumatized canine spinal cords and reveal commonalities and differences to the pathogenesis of SCI in humans. Inclusion of such a "virtual tissue bank" function into the clinical database will (1) facilitate multiinstitutional sharing of tissue available to increase the number of cases available for such basic pathological and 
molecular research and (2) provide the unique opportunity to correlate and link histopathological and molecular data to detailed clinical parameters entered in the database. While too few biological samples of any type are currently available for study, this represents an important future direction for the registry and this multi-institutional approach to tissue sharing should facilitate the accumulation of sufficient samples of all types for future study.

The current study demonstrates that prospective multiinstitutional data collection for dogs with clinical SCI is feasible and has the potential to provide valuable information to generate hypotheses and provide prevalence data important for planning future veterinary clinical trials, which can serve as a tool to enhance translational therapeutic development. It has become evident that only relying on findings from experimental rodent studies is insufficient for translating therapies to humans [40-42]. Furthermore, the impact of incorporating spontaneous animal models of disease is well-accepted in the field of cancer drug development, and rigorous multicenter veterinary clinical studies in canine spontaneous SCI have become of recent interest as a way to screen promising treatment interventions in a time- and cost-efficient manner $[16,43]$. Specifically, information gathered via the registry is useful for assessing the time between onset of injury and presentation to a veterinary SCI center, which is helpful in the planning of acute neuroprotective studies, and in assessing common comorbidities in dogs with SCI, which can aid in further development of the canine clinical model of SCI. Time required for data entry into the CSCI was minimal, with most cases requiring $6 \mathrm{~min}$ or less of data entry time. As data continue to accumulate within the registry, epidemiologic studies of this unique population of dogs with clinical SCI will become possible in order to further inform clinical studies of canine SCI. Additionally, large scale collection of data from dogs with clinical SCI may be useful for comparisons across other models and human SCI by contributing to the evolving data-sharing community, which is emerging within the field of SCI research $[18,19,44]$.

Acknowledgements This work is a product of CANSORT-SCI, the canine spinal cord injury clinical trials consortium. We gratefully acknowledge Ms. Amanda Disher, Ms. Heather Anderson, and Ms. Brittany Ridenhour for their assistance with data collection and entry.

Funding This work was funded in part by NIH UL1TR001070 and by Mission Connect, grant \# 015-105.

Author contributions S.A.M. was responsible for designing and building the database, extracting and analyzing data, interpreting data, and contributed to writing the report. N.Z. was responsible for designing and building the database and screening potentially eligible cases. She also provided feedback on the report. I.S. and Y.S.N.-L. were responsible for designing the database and contributed to writing the report. N.G., R.C.d.C., J.M.L., N.D.J., V.M.S., and A.T. were responsible for designing the database, screening potentially eligible cases, and contributed to writing the report. N.J.O. was responsible for designing the database, screening potentially eligible cases, interpreting data, and contributed to writing the report.

\section{Compliance with ethical standards}

Conflict of interest The authors declare that they have no conflict of interest.

\section{References}

1. Moore SA, Early PJ, Hettlich BF. Practice patterns in the management of acute intervertebral disc herniation in dogs. J Small Anim Pract. 2016;57:409-15.

2. Brown EA, Dickinson PJ, Mansour T, Sturges BK, Aguilar M, Young AE, et al. FGF4 retrogene on CFA12 is responsible for chondrodystrophy and intervertebral disc disease in dogs. Proc Natl Acad Sci USA. 2017;43:11476-81.

3. Smith PM, Jeffery ND. Histological and ultrastructural analysis of white matter damage after naturally-occurring spinal cord injury. Brain Pathol. 2006;16:99-109.

4. Olby N, Harris T, Burr J, Munana K, Sharp N, Keene B. Recovery of pelvic limb function in dogs following acute intervertebral disc herniations. J Neurotrauma. 2004;21:49-59.

5. Bergknut N, Egenvall A, Hagman R, Gustas P, Hazewinkel HA, Meij BP, et al. Incidence of intervertebral disk degenerationrelated diseases and associated mortality rates in dogs. J Am Vet Med Assoc. 2012;240:1300-9.

6. Frankel HL, Hancock DO, Hyslop G, Melzak J, Michaelis LS, Ungar GH, et al. The value of postural reduction in the initial management of closed injuries of the spine with paraplegia and tetraplegia. I. Paraplegia. 1969;7:179-92.

7. Olby NJ, De Risio L, Munana KR, Wosar MA, Skeen TM, Sharp NJ, et al. Development of a functional scoring system in dogs with acute spinal cord injuries. Am J Vet Res. 2001;62:1624-8.

8. Levine GJ, Levine JM, Budke CM, Kerwin SC, Au J, Vinayak A, Hettlich BF, et al. Description and repeatability of a newly developed spinal cord injury scale for dogs. Prev Vet Med. 2009;89:121-7.

9. Song RB, Basso DM, da Costa RC, Fisher LC, Mo X, Moore SA. Adaptation of the Basso-Beattie-Bresnahan locomotor rating scale for use in a clinical model of spinal cord injury in dogs. J Neurosci Methods. 2016;268:117-24.

10. Song RB, Basso DM, da Costa RC, Fisher LC, Mo X, Moore SA. von Frey anesthesiometry to assess sensory impairment after acute spinal cord injury caused by thoracolumbar intervertebral disc extrusion in dogs. Vet J. 2016;209:144-9.

11. Gorney AM, Blau SR, Dohse CS, Griffith EH, Williams KD, Lim $\mathrm{JH}$, et al. Mechanical and thermal sensory testing in normal chondrodystrophoid dogs and dogs with spinal cord injury caused by thoracolumbar intervertebral disc herniations. J Vet Intern Med. 2016;30:627-35.

12. Gutierrez-Quintana R, Edgar J, Wessman A, Cherubini GB, Penderis J. The cutaneous trunci reflex for localizing and grading thoracolumbar spinal cord injuries in dogs. J Small Anim Pract. 2012;53:470-5.

13. Jeffery ND, Smith PM, Lakatos A, Ibanez A, Ito D, Franklin RJM. Clinical canine spinal cord injury provides an opportunity to examine the issues in translating laboratory techniques into practical therapy. Spinal Cord. 2006;44:584-93.

14. Levine JM, Levine GJ, Porter BF, Topp K, Noble-Haeusslein LJ. Naturally occurring disk herniation in dogs: an opportunity for 
pre-clinical spinal cord injury research. J Neurotrauma. 2011;28:675-88.

15. Bock P, Spitzbarth I, Haist V, Stein VM, Tipold A, Puff C, et al. Spatio-temporal development of axonopathy in canine intervertebral disc disease as a translational large animal model for nonexperimental spinal cord injury. Brain Pathol. 2013;23:82-99.

16. Moore SA, Granger N, Olby NJ, Spitzbarth I, Jeffery ND, Tipold A, et al. Targeting translational successes through CANSORTSCI: using pet dogs to identify effective treatments for spinal cord injury. J Neurotrauma. 2017;34:2007-18.

17. Wewetzer K, Radtke C, Kocsis J, Baumgärtner W. Speciesspecific control of cellular proliferation and the impact of large animal models for the use of olfactory ensheathing cells and Schwann cells in spinal cord repair. Exp Neurol. 2011;229:80-7.

18. Nielson JL, Guandique CF, Liu AW, Burke DA, Lash AT, Moseanko R, et al. Development of a database for translational spinal cord injury research. J Neurotrauma. 2014;31:1789-99.

19. Callahan A, Anderson KD, Beattie MS, Bixby JL, Ferguson AR, Fouad K, et al. Developing a data sharing community for spinal cord injury research. Exp Neurol. 2017;295:135-43.

20. Wilkinson MD, Dumontier M, Aalbersberg IJJ, Appleton G, Axton M, Baak A, et al. The FAIR guiding principles for scientific data management and stewardship. Sci Data. 2016;3:160018.

21. Lemmon VP, Ferguson AR, Popovich PG, Xu X-M, Snow DM, Igarashi $\mathrm{M}$, et al. Minimum information about a spinal cord injury experiment: a proposed reporting standard for spinal cord injury experiments. J Neurotrauma. 2014;31:1354-61.

22. Biering-Sørensen F, Alai S, Anderson K, Charlifue S, Chen Y, DeVivo M, et al. Common data elements for spinal cord injury clinical research: a National Institute for Neurological Disorders and Stroke project. Spinal Cord. 2015;53:265-77.

23. Charlifue S, Tate D, Biering-Sorensen F, Burns S, Chen Y, Chun S, et al. Harmonization of databases: a step for advancing the knowledge about spinal cord injury. Arch Phys Med Rehabil. 2016;97:1805-18.

24. Tarlov IM, Klinger H. Spinal cord compression studies. II. Time limits for recovery after acute compression in dogs. AMA Arch Neurol Psychiatry. 1954;71:271-90.

25. Harris P, Taylor R, Thielke R, Payne J, Gonzalez N, Conde JG. Research electronic data capture (REDCap) - a metadata-driven methodology and workflow process for providing translational research informatics support. J Biomed Inform. 2009;42:377-81.

26. Marin J, Nixon J, Gorecki C. A systematic review of risk factors for the development and recurrence of pressure ulcers in people with spinal cord injuries. Spinal Cord. 2013;51:522-7.

27. van der Scheer JW, Martin Ginis KA, Ditor DS, Goosey-Tolfrey VL, Hicks AL, West CR, et al. Effects of exercise on fitness and health of adults with spinal cord injury: a systematic review. Neurology. 2017;89:736-45.

28. American Animal Hospital Association. Body condition scoring (BCS) systems. 2010. https://www.aahanet.org/PublicDocuments/ NutritionalAssessmentGuidelines.pdf. Accessed 16 August 2017.

29. McGreevy PD, Thomson PC, Pride C, Fawcett A, Grassi T, Jone B. Prevalence of obesity in dogs examined by Australian veterinary practices and the risk factors involved. Vet Rec. 2005;156:695-702.
30. Lund EM, Armstrong PJ, Kirk CA, Klausner JS. Prevalence and risk factors for obesity in adult dogs from private US veterinary practices. Intern J Appl Res Vet Med. 2009;4:177-86.

31. Gayet C, Bailhache E, Dumon H, Martin L, Siliart B, Nguyen P. Insulin resistance and changes in plasma concentration of $\mathrm{TNF} \alpha$, IGF1, and NEFA in dogs during weight gain and obesity. J Anim Physiol Anim Nutr. 2004;88:157-65.

32. Park HJ, Lee SE, Kim HB, Isaacson RE, Seo KW, Song KH. Association of obesity with serum leptin, adiponectin, and serotonin and gut microflora in beagle dogs. J Vet Intern Med. 2015;29:43-50.

33. Mazaki-Tovi M, Bolin SR, Schenck PA. Differential secretion of adipokines from subcutaneous and visceral adipose tissue in healthy dogs: association with body condition and response to troglitazone. Vet J. 2016;216:136-41.

34. Stenson KW, Deutsch A, Heinemann AW, Chen D. Obesity and inpatient rehabilitation outcomes for patients with traumatic spinal cord injury. Arch Phys Med Rehabil. 2011;92:384-9.

35. Kigerl KA, Hall JCE, Wang L, Mo X, Yu Z, Popovich PG. Gut dysbiosis impairs recovery after spinal cord injury. J Exp Med. 2016;213:2603-20.

36. Aupperle H, Marz I, Theilebein J, Kiefe B, Kappe A, Schoon HA. Immunohistochemical characterization of the extracellular matrix in normal mitral valves and in chronic valve disease (endocardiosis) in dogs. Res Vet Sci. 2009;87:277-83.

37. Boswood A, Haggstrom J, Gordon SG, Wess G, Stepien RL, Oyama MA, et al. Effect of pimobendan in dogs with preclinical myxomatous mitral valve disease and cardiomegaly: the EPIC study - a randomized clinical trial. $\mathrm{J}$ Vet Intern Med. 2016;30:1765-79.

38. Olby N, Levine J, Harris T, Munana K, Skeen T, Sharp N. Longterm functional outcome of dogs with severe injuries of the thoracolumbar spinal cord: 87 cases (1996-2001). J Am Vet Med Assoc. 2003;222:762-9.

39. Granger N, Carwardine D. Acute spinal cord injury: tetraplegia and paraplegia in small animals. Vet Clin North Am Small Anim Pract. 2014;44:1131-56.

40. Rosenzweig ES, Courtine G, Jindrich DL, Brock JH, Ferguson AR, Strand SC, et al. Extensive spontaneous plasticity of corticospinal projections after primate spinal cord injury. Nat Neurosci. 2010;13:1505-10.

41. Nout YS, Rosenzweig ES, Brock JH, Strand SC, Moseanko R, Hawbecker S, et al. Animal models for neurologic disorders: a non-human primate model of spinal cord injury. Neurotherapeutics. 2012;9:380-92.

42. Rosenzweig ES,Brock JH,Lu P,Hiromi K,Salegio EA,Kadoya K, et al. Restorative effects of human neural stem cell grafts on the primate spinal cord. Nat Med. 2018;24:484-90.

43. Gordon I, Paoloni M, Mazcko C, Khanna C. The comparative oncology trials consortium: using spontaneously occurring cancers in dogs to inform the dance drug development pathway. PLoS Med. 2009;6:e1000161.

44. Nielson JL, Paquette J, Liu AW, Guandique CF, Tovar CA, Inoue $\mathrm{T}$, et al. Topographical data analysis for discovery in preclinical spinal cord injury and traumatic brain injury. Nat Commun. 2015;6:8581.

\title{
Affiliations
}

\author{
Sarah A. Moore ${ }^{1} \cdot$ Natalia Zidan $^{2} \cdot$ Ingo Spitzbarth $^{3,4} \cdot$ Yvette S. Nout-Lomas $^{5} \cdot$ Nicolas Granger $^{6}$. \\ Ronaldo C. da Costa ${ }^{1} \cdot$ Jonathan M. Levine ${ }^{7}$ Nick D. Jeffery ${ }^{7} \cdot$ Veronika M. Stein $^{8} \cdot$ Andrea Tipold $^{4,9}$. \\ Natasha J. Olby ${ }^{2,10}$
}


1 Department of Veterinary Clinical Sciences, The Ohio State University College of Veterinary Medicine, Columbus, OH, USA

2 Department of Clinical Sciences, College of Veterinary Medicine, North Carolina State University, Raleigh, NC, USA

3 Department of Pathology, University of Veterinary Medicine Hannover, Hannover, Germany

4 Center for Systems Neuroscience, Hannover, Germany

5 College of Veterinary Medicine and Biomedical Sciences, Colorado State University, Fort Collins, CO, USA

6 Department of Small Animal Clinical Sciences, School of
Veterinary Sciences, University of Bristol, College of Veterinary Medicine and Biomedical Sciences, Langford, UK

7 Texas A \&M University, College Station, TX, USA

8 Department of Clinical Veterinary Sciences, University of Bern, Bern, Switzerland

9 Department of Small Animal Medicine and Surgery, University of Veterinary Medicine, Hannover, Germany

10 Comparative Medicine Institute, North Carolina State University, Raleigh, NC, USA 